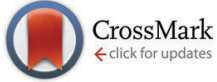

Cite this: New J. Chem., 2015, 39, 5895

Received (in Montpellier, France) 23rd April 2015,

Accepted 29th May 2015

DOI: 10.1039/c5nj01011j

www.rsc.org/njc

\title{
Sputtering synthesis and optical investigation of octadecanethiol-protected fluorescent $\mathrm{Au}$ nanoparticles $\uparrow$
}

\author{
Yohei Ishida, Taiki Sumi and Tetsu Yonezawa*
}

\begin{abstract}
Herein we report for the first time the synthesis of octadecanethiolcapped gold nanoparticles (Au NPs) by sputtering of Au over a liquid matrix (silicone oil). Au NPs prepared in silicone oil showed plasmon absorption; however, those prepared in the presence of 1-octadecanethiol did not show plasmon absorption but fluoresced in the near IR region.
\end{abstract}

Metal nanoparticles (NPs) are attracting considerable interest due to a large number of potential applications resulting from both high surface-to-bulk ratio and quantum confinement of electrons localized inside NPs. ${ }^{1-4}$ Various preparation methods for metal NPs have been investigated but, recently, sputtering deposition over liquid matrices has been intensively studied in order to generate stable colloidal metal NPs. ${ }^{5,6}$ This synthetic method takes advantage of the extremely low vapor pressure of liquid matrices so that the preparation of NPs can be carried out at low pressure or under high vacuum conditions. In a sputtering process, ionized argon at high voltage attacks the target metal and ejects the target atoms or atom clusters into a vacuum chamber. Ejected atoms and clusters coalesce into NPs in the gas phase or at the interface of the liquid matrix. In the past, several liquid matrices have been examined including ionic liquids, ${ }^{5,7}$ propane-1,2,3-triol, ${ }^{8}$ pentaerythritol ethoxylate, ${ }^{9}$ polyethylene glycol (PEG), ${ }^{10-12}$ 6-mercaptohexyl-trimethylammoniumbromide, ${ }^{6}$ and pentaerythritol tetrakis(3-mercaptopropionate). ${ }^{9}$ All these liquid matrices were able to produce $\mathrm{Au}$ NPs with approximately $10 \mathrm{~nm}$ diameter. The last two matrices listed above, however, produced rather small NPs with diameters of approximately 1-2 $\mathrm{nm}$. This phenomenon was attributed to the coordination of mercapto groups in the liquid matrix molecules to the Au NP surface that prevented the aggregation and

Division of Material Science and Engineering, Faculty of Engineering,

Hokkaido University, Kita 13, Nishi 8, Kita-ku, Sapporo, Hokkaido 060-8628, Japan. E-mail: tetsu@eng.hokudai.ac.jp

$\dagger$ Electronic supplementary information (ESI) available: Detailed experimental procedures. See DOI: 10.1039/c5nj01011j

growth of Au NPs. The Au NPs obtained in such "thiolate matrices" fluoresced due to their very small particle sizes hence plasmon absorption was negligible. Very recently, we reported the synthesis of fluorescent $\mathrm{Au}$ and $\mathrm{Ag}$ NPs by sputtering onto thiolate-containing PEG. In this procedure, we successfully controlled the diameter of NPs by changing the concentration of thiolate ligands. ${ }^{11,12}$ This approach can be expanded to other combinations of liquid matrices and thiolate ligands, and will broaden the spectrum of NP chemistry using this sputtering technique.

We herein present a novel set of fluorescent Au NPs synthesized by sputtering onto liquid matrices (silicone oil: Si-oil) containing alkylthiol (1-octadecanethiol: ODT). This method aims at the stabilization of Au NPs by ODT both inside and at the interface of the Si-oil. It is expected that the concentration of ODT directly controls the diameter of Au NPs to single nanometer sizes. Alkylthiol is usually very volatile and not suitable for matrix sputtering, but ODT can be used for this purpose due to its high boiling point.

Three different matrix compositions were studied in this work: (a) Si-oil, (b) $3.6 \times 10^{-3} \mathrm{M}$ ODT in Si-oil, and (c) molten ODT (3.0 M). Extinction spectra of Au NP dispersions were measured in a quartz cell with $1 \mathrm{~mm}$ optical path just after sputtering (Fig. 1). Samples (a) and (b) were measured without dilution, and sample (c) was measured after a 1:5 dilution with acetonitrile ( 1 vol. part ODT: 5 vol. parts acetonitrile). A broad absorption peak at around $520 \mathrm{~nm}$ was observed for sample (a). This peak is usually observable for Au NPs and corresponds to the localized plasmon absorption of $\mathrm{Au}$. On the other hand, samples (b) and (c) did not show any plasmon absorption peaks in their absorption spectra. The results for (b) and (c) may be due to the difference in the sizes of these NPs. Surface plasmon absorption generally arises from a vibration of free electrons on the surface of NPs and so shows an absorption corresponding to these vibration frequencies. Plasmon absorption thus requires a certain particle size. ${ }^{13,14}$ When the number of atoms in each particle decreases, the energy band gap becomes wider according to the quantum size effect and a particle becomes non-metallic. 


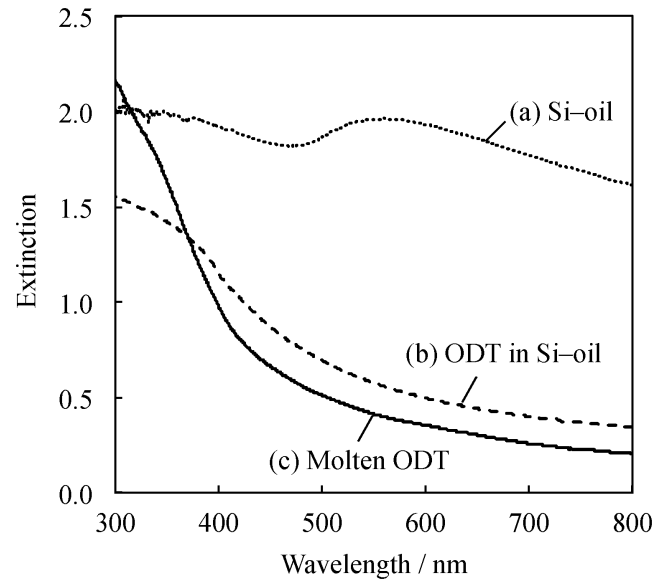

Fig. 1 Extinction spectra of Au NPs prepared by sputtering onto (a) Si-oil, (b) $3.6 \times 10^{-3} \mathrm{M}$ ODT in Si-oil, and (c) molten ODT. The spectra were measured in a $1 \mathrm{~mm}$ quartz cell without dilution for (a) and (b), and a 1:5 dilution with acetonitrile for (c).
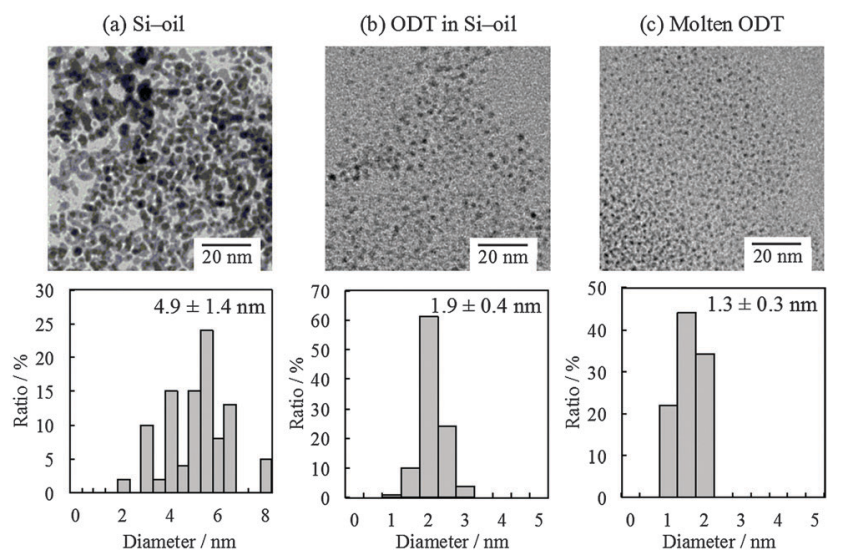

Fig. 2 TEM images and size-distribution histograms of Au NPs prepared by sputtering onto (a) Si-oil, (b) $3.6 \times 10^{-3} \mathrm{M}$ of ODT in Si-oil, and (c) molten ODT. The histogram data were prepared from 200 NPs counted in more than 5 TEM images.

Thus, plasmon absorption is not observable in particles less than $2 \mathrm{~nm}$ in diameter for Au. ${ }^{15}$ Judging from the absence of plasmon absorption in Fig. 1, very small particle diameters are expected for samples (b) and (c).

Transmission electron microscopy (TEM) was used to determine the particle diameters. Fig. 2 shows representative TEM images and size-distribution histograms. The average sizes of the Au NPs were determined to be $4.9 \pm 1.4 \mathrm{~nm}, 1.9 \pm 0.4 \mathrm{~nm}$, and $1.3 \pm 0.3 \mathrm{~nm}$ for samples (a)-(c), respectively. As we determined from the extinction spectra in Fig. 1, the diameter of sample (a) was significantly larger than that of (b) and (c). Moreover, the average NP diameter for samples (b) and (c) was quite different. This could have originated from the suppression of the coalescence of NPs on or in the matrix. The concentration of ODT in sample (b) was $3.6 \times 10^{-3} \mathrm{M}$, and the concentration of molten ODT for sample (c) was 3.0 M. Thus, the difference in ODT concentration in these two samples affected the collision probability between ODT and the Au NPs, which may be the reason for such a difference in their sizes.
From these TEM images, we can recognize the inter-particle interactions in the current system. ODT-capped Au NPs formed in this study were not soluble in common organic solvents such as chloroform, hexane, and dimethylsulfoxide. In contrast, ODTcapped Au NPs prepared by the chemical reduction method were quite soluble in these solvents. ${ }^{16}$ The chain length of ODT is approximately $2 \mathrm{~nm},{ }^{17}$ thus the distance between adjacent $\mathrm{Au}$ NPs should be larger than $4 \mathrm{~nm}$ when the Au NPs exist as mononanoparticles without subsequent aggregation. From Fig. 2c, the average inter-particle distance was calculated to be $2.1 \mathrm{~nm}$ (see the histogram of edge-to-edge inter-particle distances shown in Fig. S1, ESI $\dagger$ ). Thus, it is obvious that the long hydrophobic alkyl chains in ODT were entangled between 2 or more nanoparticles. The insolubility of these Au NPs likely resulted from this phenomenon. This entanglement of the surface ligands may be attributed to the very high concentration of ODT $(3.0 \mathrm{M})$ and their long hydrophobic alkyl chains.

The absence of plasmon absorption and the very small particle sizes of samples (b) and (c) led us to study the fluorescence properties of Au NPs stabilized by ODT. The excitation wavelength was set at $300 \mathrm{~nm}$. Sample (a), which shows plasmon absorption in Fig. 1, did not fluoresce. On the other hand, samples (b) and (c) fluoresced in the near IR region. Fig. 3 shows a fluorescence spectrum of sample (c) (also see sample pictures under sunlight and UV-irradiation in Fig. S2, ESI $\dagger$ ). The spectral shapes of samples (b) and (c) were similar but the fluorescence maxima $\left(\lambda_{\mathrm{Fl}}\right)$ were slightly different from each other. The $\lambda_{\mathrm{Fl}}$ were 677 and $664 \mathrm{~nm}$ for samples (b) and (c), respectively. As we previously reported, in the matrix sputtering synthesis of Au NPs, the fluorescence maxima tend to shift to the red as the average diameter of the nanoparticles increases. ${ }^{11,12}$ This phenomenon is attributed to the quantum size effect, ${ }^{18}$ in which a band gap of NPs depends on the number of atoms making up a particle.

The excitation spectrum of sample (c) was observed at the fluorescence maximum (664 nm) as shown by the dashed line in Fig. 3. The excitation maximum was observed at $356 \mathrm{~nm}$; therefore, the Stokes shift for these Au NPs was determined to

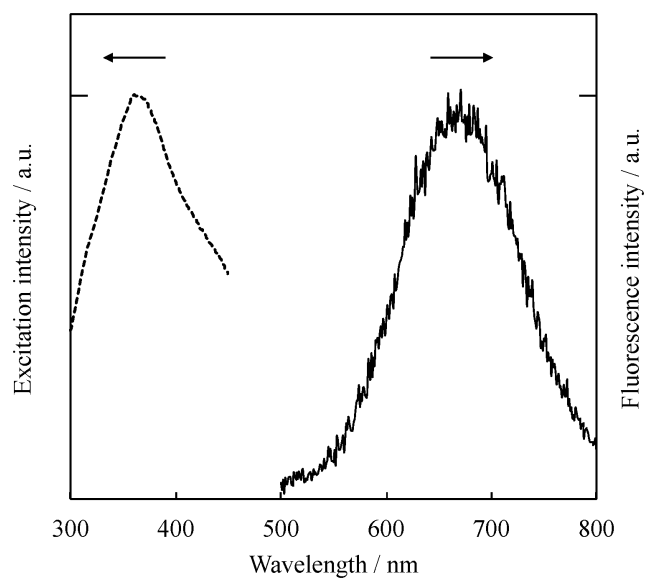

Fig. 3 Excitation and fluorescence spectra of Au NPs prepared by sputtering onto molten ODT (sample c). The excitation wavelength was set at $300 \mathrm{~nm}$, and the excitation spectrum was observed at $664 \mathrm{~nm}$. 
be $1.62 \mathrm{eV}$. This large Stokes shift was reported in our previous matrix sputtering synthesis work. The main reason for this shift was considered to be the stabilization of the d band and/or the destabilization of the sp-conduction band in the Au NPs, which usually results in a blue shift in the absorption (excitation) spectrum. ${ }^{6}$

The fluorescence quantum yields of samples (b) and (c) were measured by the absolute method. Both samples showed a $1.0 \%$ quantum yield. These values were similar to those of gold nanoparticles and nanoclusters prepared by various other synthetic methods including sputtering and chemical reduction methods. ${ }^{19,20}$

In conclusion, we have reported the synthesis of octadecanethiolcapped fluorescent Au NPs by sputtering over silicone oil. The photophysical characteristics were fully investigated by absorption and fluorescence measurements combined with TEM. The sputtering method presented here can be expanded to a wide variety of liquid matrix-thiolate ligand combinations. Hence, it is expected to be a novel synthetic candidate for functional metal NPs with precisely controlled diameters and photophysical characteristics.

\section{Experimental section}

The liquid matrix used in this work was a silicone oil obtained from Shinetsu (Si-oil, KF-96-50cs, kinematic viscosity of $50 \mathrm{~mm}^{2} \mathrm{~s}^{-1}$ at $25{ }^{\circ} \mathrm{C}$ ) that is often used in synthetic baths. 1-Octadecanethiol (ODT, Wako) was used as the alkylthiol stabilizer for Au NPs. An $\mathrm{Au}$ target (99.9\%) was supplied by Tanaka Precious Metals (Japan). The experimental procedure is described below. First, in order to remove any volatile substances, Si-oil and ODT were dried under vacuum at $100{ }^{\circ} \mathrm{C}$ for $2 \mathrm{~h}$. Three different capturing matrix compositions were used in this work: (a) $1.0 \mathrm{~g}$ of Si-oil, (b) $1.0 \mathrm{~g}$ of Si-oil containing $10 \mathrm{mg}$ of ODT (corresponding to $3.6 \times 10^{-3} \mathrm{M}$ ODT in Si-oil), and (c) $1.0 \mathrm{~g}$ of molten ODT (corresponding to $3.0 \mathrm{M}$ ).

For the sputtering syntheses, solutions (a)-(c) were added separately into glass Petri dishes having a diameter of $30 \mathrm{~mm}$ and were horizontally positioned against the sputtering target. $\mathrm{Au}$ was sputtered at a current of $30 \mathrm{~mA}$ under Ar and the pressure was kept at $20 \mathrm{~Pa}$. The sputtering voltage was ca. $200 \mathrm{~V}$. The distance between the surface of PEG and the surface of the gold target was $25 \mathrm{~mm}$. Sputtering was carried out for $30 \mathrm{~min}$ at $50{ }^{\circ} \mathrm{C}$ under stirring at $100 \mathrm{rpm}$. Due to the temperature inside the sputtering chamber, ODT completely dissolved in Si-oil at the concentration prepared for sample (b) and was completely liquefied in sample (c). The experimental details for the characterization and observation for the resulting NPs are described in the ESI. $\dagger$

\section{Acknowledgements}

This work was partially supported by Hokkaido University and Grant-in-Aid for Scientific Research (A) (to TY 24241041), and by
Building of Consortia for the Development of Human Resources in Science and Technology (to YI). This work was also partially supported by Hokkaido University, microstructural characterization platform as a program of Nanotechnology Platform of the Ministry of Education, Culture, Sports, Science and Technology (MEXT), Japan.

\section{Notes and references}

1 N. Toshima and T. Yonezawa, New J. Chem., 1998, 22, 1179.

2 D. V. Talapin, J.-S. Lee, M. V. Kovalenko and E. V. Shevchenko, Chem. Rev., 2010, 110, 389.

3 R. L. Whetten and R. C. Price, Science, 2007, 318, 407.

4 E. V. Shevchenko, D. V. Talapin, N. A. Kotov, S. O'Brien and C. B. Murray, Nature, 2006, 439, 55.

5 T. Torimoto, K.-I. Okazaki, T. Kiyama, K. Hirahara, N. Tanaka and S. Kuwabata, Appl. Phys. Lett., 2006, 89, 243117.

6 Y. Shishino, T. Yonezawa, K. Kawai and H. Nishihara, Chem. Commun., 2010, 46, 7211.

7 H. P. S. Castro, H. Wender, M. A. R. C. Alencar, S. R. Teixeira, J. Dupont and J. M. Hickmann, J. Appl. Phys., 2013, 114, 183104.

8 J. Siegel, O. Kvítek, P. Ulbrich, Z. Kolská, P. Slepička and V. Švorčík, Mater. Lett., 2012, 89, 47.

9 Y. Shishino, T. Yonezawa, S. Udagawa, K. Hase and H. Nishihara, Angew. Chem., Int. Ed., 2010, 50, 703.

10 Y. Hatakeyama, T. Morita, S. Takahashi, K. Onishi and K. Nishikawa, J. Phys. Chem. C, 2011, 115, 3279.

11 T. Sumi, S. Motono, Y. Ishida, N. Shirahata and T. Yonezawa, Langmuir, 2015, 31, 4323.

12 Y. Ishida, R. Nakabayashi and T. Yonezawa, New J. Chem., 2015, 39, 4227.

13 N. Schaeffer, B. Tan, C. Dickinson, M. J. Rosseinsky, A. Laromaine, D. W. McComb, M. M. Stevens, Y. Wang, L. Petit, C. Barentin, D. G. Spiller, A. I. Cooper and R. Lévy, Chem. Commun., 2008, 3986.

14 S. H. Yau, O. Varnavski and T. Goodson, Acc. Chem. Res., 2013, 46, 1506.

15 C. Kumara, X. Zuo, D. A. Cullen and A. Dass, ACS Nano, 2014, 8, 6431.

16 A. Badia, L. Cuccia, L. Demers, F. Morin and R. B. Lennox, J. Am. Chem. Soc., 1997, 119, 2682.

17 N. A. Ray, R. P. Van Duyne and P. C. Stair, J. Phys. Chem. C, 2012, 116, 7748.

18 J. A. McLean, K. A. Stumpo and D. H. Russell, J. Am. Chem. Soc., 2005, 127, 5304.

19 P. Yu, X. Wen, Y.-R. Toh and J. Tang, J. Phys. Chem. C, 2012, 116, 6567.

20 T. Chen, S. Xu, T. Zhao, L. Zhu, D. Wei, Y. Li, H. Zhang and C. Zhao, ACS Appl. Mater. Interfaces, 2012, 4, 5766. 